\title{
To determine the optimal size of the elements of complex overlap
}

\author{
Vitaly Kuznetsov and Yulia Shaposhnikova* \\ Moscow State University of Civil Engineering, Yaroslavskoe shosse, 26, Moscow, 129337, Russia
}

\begin{abstract}
The article discusses the possibilities of optimizing the size of elements of reinforced concrete ceilings along. The optimization of the overlap consists in finding such parameters of the reinforced concrete slab, profiled flooring and steel beams, in which their strength is fully used. For ceilings with steel beams included, the dimensions of the sections of the elements and their strength characteristics are determined by the requirements of the limit states of the second group (deflections), and the calculation of the forces in the slab and beams and strength calculations are performed taking into account the geometric and strength characteristics established from the calculation of deformations. To clarify the features of the calculation and verification of the main provisions, a numerical experiment was carried out, where the center cell of the steel concrete slab along metal rolling beams was selected as the object of study. Formulas are obtained for determining the forces using predetermined dimensions of the components of the overlap based on satisfying the requirements of the 2 groups of limit states. These studies can serve as a basis for further research on the search for optimal parameters of complex floors.
\end{abstract}

\section{Introduction}

Complex monolithic reinforced concrete floors consist of steel beams, profiled flooring and reinforced concrete slabs (Figure1). In such ceilings, the profiled sheet is a formwork system that provides the structure with additional rigidity, which makes it possible to create a cost-effective and easily constructed structure, therefore the search for the optimal dimensions of such structures is always relevant [1-3].

\footnotetext{
* Corresponding author: yuliatalyzova@yandex.ru
} 


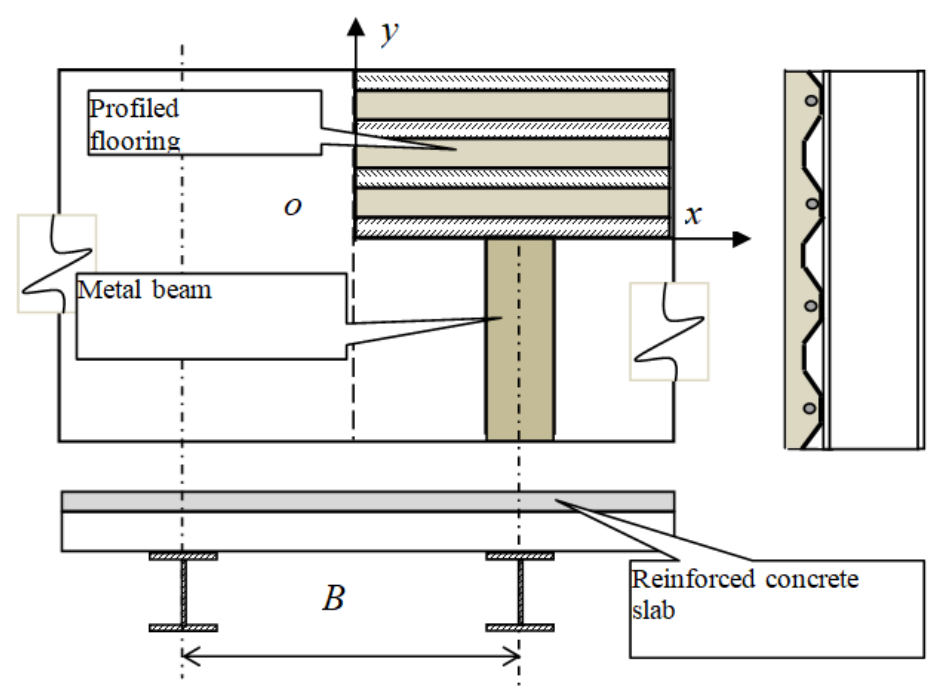

Fig. 1. Overlap scheme

Many Russian and foreign scientists were studying the work of complex monolithic ceilings, supported on metal beams. For example, in [1-3] the questions of the relevance of the use of such structures in buildings of various purposes have been studied. Features of calculations of such overlaps are presented in the studies [4-16].

Traditionally, the calculation of strength was carried out in accordance with the distribution of forces, in proportion to the rigidity of the components of the overlap [17]. Since 1984, the calculation of composite structures has been carried out in accordance with [18] and its subsequent versions [19], and in 2017 a normative document [20] has been introduced, which to some extent duplicates the provisions of previous standards. At present, in Russia it is recommended to calculate the steel-reinforced concrete structures in accordance with [20].

\section{Methods}

A feature of the calculation of these structures is to take into account the joint work of the profiled flooring with a reinforced concrete slab and steel beams. The connection of profiled flooring with a reinforced concrete slab, as a rule, is ensured by reliable adhesion of the decking and concrete slab, due to constructive measures and can be considered as a reinforced concrete slab with anisotropic stiffness. The retaining of a steel concrete slab on steel beams, as a rule, is a hinge and the distribution of forces from vertical loads is proportional to the flexural rigidity of the constituent elements.

In general, the bearing capacity of the slab (external bending moment $M_{p l}$ ) depends on the thickness of the concrete slab $h_{p l}$, the concrete strength of the slab $R_{b}$, the strength of the reinforcement of the slab $R_{s}$, the amount of reinforcement of the slab $A_{s}$, the cross-sectional dimensions $A$ and the strength of the sheeting $R_{s, p f}$

$$
M_{p l}=M\left(h_{p l} ; R_{b} ; R_{s} ; A_{s} ; A ; R_{s, p f}\right)
$$

The bearing capacity of the steel beam $\left(M_{b}\right)$ is a function of the geometrical parameters of the section and, in particular, the moment of resistance $W_{b}$ and strength of the material $R_{s b}$.

$$
M_{b}=M\left(W_{b} ; R_{s b}\right)
$$


Thus, the overlap strength in general $(\mathrm{M})$ is determined by eight variable parameters, reflecting the geometric characteristics of the cross sections and the strength properties of materials.

$$
M=M\left(h_{p l} ; R_{b} ; R_{s} ; A_{s} ; A ; R_{s, p f} ; R_{s b} ; W_{b}\right)
$$

The optimization of the overlap consists in finding such parameters of the reinforced concrete slab, profiled flooring and steel beams for which their strength is used completely (the utilization rate of materials is close to unity).

However, in most cases, for ceilings with steel beams included, the dimensions of the sections of elements and their strength characteristics are determined by the requirements of the limiting states of the second group (deflections), and the calculation of forces in the slab and beams and strength calculations are performed taking into account the geometric and strength characteristics, established from the calculation of deformations.

To clarify the features of the calculation and verification of the main provisions, a numerical experiment was conducted, where the central cell of the steel concrete slab along metal rolling beams was selected as the object of study (Fig. 1).

In case of articulated support of the slab along the corrugated wave and uniform load, the maximum deflection in the middle of the span, in the slab stage of operation without cracks and without regard to the rigidity of the flooring, is equal to

$$
f_{p l}=\frac{5 q_{p l} l_{p l}^{4}}{384 E_{p l} I_{p l}}
$$

$q_{p l}$ - linear load on the slab, including dead weight,

$l_{\mathrm{pl}}$ - the calculated span of the slab was taken to be equal to the distance between the extreme supports, and the influence of the local deflection between the beams was not taken into account due to their smallness.

When $n_{p l}=l_{p l} / f_{p l}$ the moment of inertia of the plate is equal to

$$
I_{p l}=\frac{5 n_{p l} q_{p l} l_{p l}^{3}}{384 E_{p l}}
$$

At the moment of inertia $I_{p l}=b_{p l} h_{p l}{ }^{3} / 12, b=1$ the height of the plate section is equal to

$$
h_{p l}=\frac{l_{p l}}{4} \sqrt[3]{\frac{q_{p l} n}{E_{p l}}}
$$

Here, the coefficient of relative deflection $n=l_{p l} / f_{p l}$.

\section{Results and discussion}

In Figure 2 graphs of slab height under short-term load action $\left(l_{p l}=600 \mathrm{~cm}, n=200\right.$, concrete class B20) are presented. The graphs show that the requirement of the plate rigidity, has a greater influence on the choice of height, than load growth. 


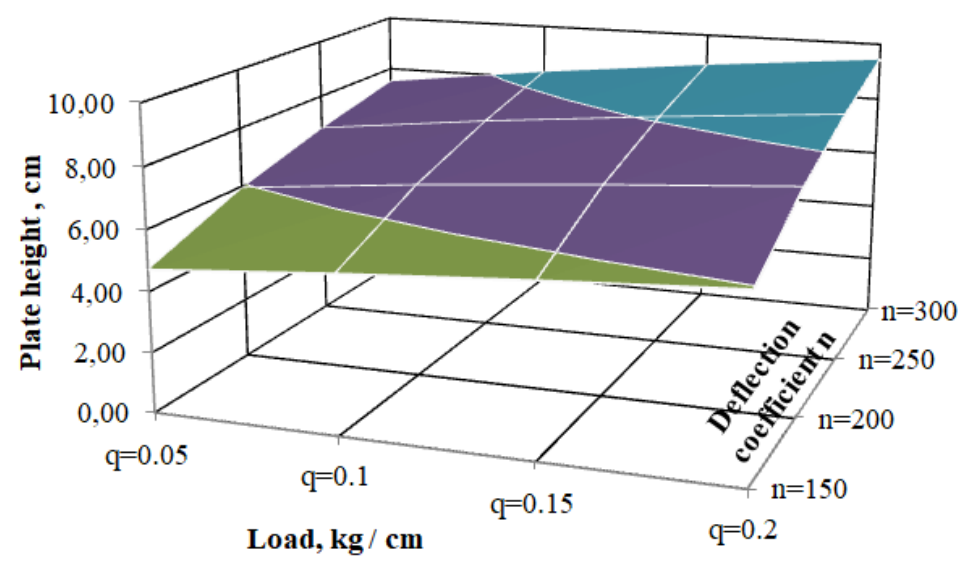

Fig. 2. The dependence of the height of the plate from the load $q$ and $n$

Similarly, the moment of inertia of the beam is

$$
I_{b}=\frac{5 n_{b} q_{b} l_{b}^{3}}{384 E_{b}}
$$

$q_{b}$ - full regulatory load on the beam, including its own weight,

$l_{b}$ - the estimated span of the beam,

coefficient of relative deflection $n_{b}=l_{b} / f_{b}$.

In Figure 3 graphs of inertia moments change under short-term load action are presented (beam span $l_{b}=600 \mathrm{~cm}, n=200$, distance between beams $B=1 \mathrm{~m}$ ).

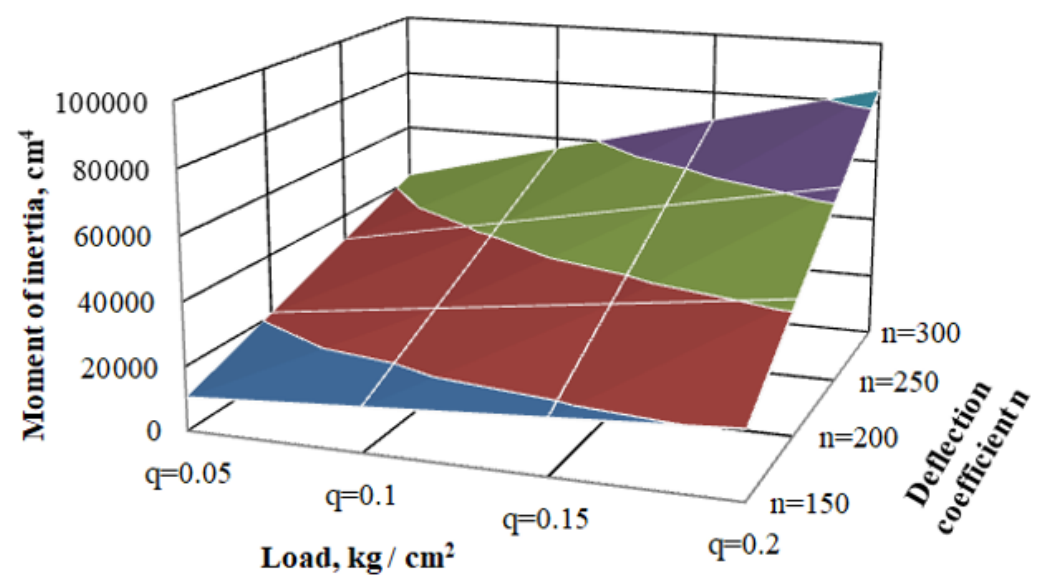

Fig. 3. Dependence of the moment of inertia $I=I(n ; q)$

To derive general patterns of calculations, we consider a profiled sheet and a reinforced concrete slab as working together, that is, a single, seamless structure, where the distribution of forces should be proportional to the stiffness of the beam and floor, calculated from the calculation of equality based on the limiting deflections. 


$$
\frac{M_{p l}}{M_{b}}=\frac{I_{p l}}{I_{b}}=\frac{384 E_{b}}{5 n_{b} q_{b} l_{b}^{3}} \cdot \frac{5 n_{p l} q_{p l} l_{p l}^{3}}{384 E_{p l}}=\frac{E_{b}}{E_{p l}} \cdot \frac{n_{p l} q_{p l} l_{p l}^{3}}{n_{b} q_{b} l_{b}^{3}}
$$

When replacing the ratio of the modulus of elasticity of concrete slabs to the modulus of elasticity of steel beams by the coefficient of reduction $\alpha$

$$
\frac{M_{p l}}{M_{b}}=\alpha \cdot \frac{n_{p l} q_{p l} l_{p l}^{3}}{n_{b} q_{b} l_{b}^{3}}
$$

Taking into account that the load on the beam can be expressed through the load on the slab and the step of the beams $q b=\mathrm{V} \cdot q p l$

$$
\frac{M_{p l}}{M_{b}}=\alpha \cdot \frac{n_{p l} l_{p l}^{3}}{n_{b} B l_{b}^{3}}
$$

In the special case when the beam span equal span of end support plates $l_{b}=l_{p l}$, formulas (9) and (10) take the form respectively

$$
\frac{M_{p l}}{M_{b}}=\alpha \cdot \frac{q_{p l}}{q_{b}} \quad \text { и } \quad \frac{M_{p l}}{M_{b}}=\frac{\alpha}{B}
$$

Since 2017, the design of such ceilings, in the Russian Federation used the joint venture 266.1325800. 2016 «Structures of steel reinforced concrete. Design rules» [20].

\section{Conclusions}

1. These formulas can be used to define forces using predetermined dimensions of the components of the overlap based on satisfying the requirements of the 2 groups of limit states.

2. The presented article can serve as a basis for further research on the search for optimal parameters of complex floors.

\section{References}

1. V.S. Babalich, E.N. Androsov Steel-reinforced concrete structures and the prospect of their application in the construction practice of Russia. The successes of modern science. 4, 205-208 (2017)

2. P.I. Egorov, S.A. Korolev Steel reinforced concrete floors Far East: the problems of the development of the architectural and construction complex. 1, 310-313 (2015)

3. A.G. Tamrazyan, S.N. Arutyunyan To the assessment of the reliability of steel slabs of slabs with profiled floorings Herald of Civil Engineers. 6, 52-57 (2015)

4. A.N. Klimov Designing composite structures for euronorms Concrete technology. 5-6, 57-59 (2015)

5. F.S. Zamaliev Consideration of nonlinear properties of materials and pliability of layers when calculating the strength of steel concrete slabs Industrial and civil engineering. 5, 38-40 (2013)

6. N.G, Gonshakov, A.G. Gonshakov, M.S. Sergeyev, M.O. Lisyatnikova Determination of capacity of the plate of monolithic reinforced concrete with steel profiled floor, working in two directions Bulletin of construction equipment 12 (1000), 48-49 (2017) 
7. G.M. Bazhin Analysis of the tensive status, when the beam covering is included including the joint work of the profiled floor International scientific journal. 6, 61-65 (2017)

8. V.S. Kuznetsov, Yu.A. Shaposhnikova, K.A. Budoshkina, Yu. A. Murlisheva, A.S. Ulyamaev Strengthening of reinforeced concrete floors with pre-stressed rebars Engineering Journal of Don. 2 (2018)

9. V. Cherednikov, O. Voskobiinyk, O. Cherednikova Evaluation of the warping model for analysis of polystyrene concrete slabs with profiled steel sheeting Periodica polytechnica: civil engineering. 61, 3, 483-490 (2017)

10. I. A. Rumyantseva Steel profiled in steel line concrete reinsures Transport construction. 4, 28 (2009)

11. V.S. Kuznetsov, Yu.A. Shaposhnikova, K.A. Budoshkina, Yu. A. Murlisheva, A.S. Ulyamaev Analysis of the operation of combined beams in a wide range of loads Engineering Journal of Don. 2 (2018)

12. E.L. Ayrumyan, N.I. Kamenshchikov, I.A. Rumyantseva Features of the calculation of monolithic slabs of composite slabs on profiled steel flooring Industrial and civil engineering. 9, 21-26 (2015)

13. S.O. Postanen, A.Yu. Berezkina, V.V. Komissarov, M.O. Postanen Steel reinforced concrete floors on profiled steel flooring Young Scientist. 26, 74-76 (2016)

14. A.G. Tamrazyan, S.N. Arutyunyan To the account of profiled flooring as working reinforcement in the calculation of monolithic reinforced concrete slabs for overcoating Industrial and civil engineering. 7, 64-68 (2016)

15. F.S. Zamaliev To the calculation of steel slabs with steel profiled flooring News of Kazan State Architectural and Construction University. 3, 129-134 (2016)

16. A.M. Zulpuev., K. Baktygulov Two-dimensional model of calculation of reinforcedconcrete composite beams by the method of concentrated deformations ARPN Journal of engineering and applied sciences. 4, 1030-1037 (2017)

17. A.A. Umansky Directory of the designer of industrial, residential and public buildings and structures. Calculated-theoretical M., State publishing house of literature on construction, architecture and building materials. (1960)

18. SNiP 35.13330.2011 Bridges and pipes (1984)

19. SNiP 35.13330.2011 Bridges and pipes (2011)

20. SP 266.1325800.2016 Structures of steel reinforced concrete. Design rules (2016) 